\title{
Lustrum, the oldest relays of the Tor network and their ISP's : more data.
}

\author{
Camille Akmut*
}

June 12, 2019

\begin{abstract}
A quantitative approach to a historical problem. Sister publication to "Fearless, 1000 days and still running : the 'most resilient' exit nodes of the Tor network".
\end{abstract}

Keywords : Tor; network resilience; network diversity; ISP; metrics.

*melchior@nerv 


\section{Methodology}

We looked this time at the oldest relays within the Tor network.

Here all relays were considered : whether exit nodes, entry guards, or else. $^{1}$

Our criteria were : age (5 years, or 1825 days, of minimum existence) and present status ('running').

Using the Tor metrics database, still, our search query was thus :

running:true first_seen_days:1825-10000

With 556 matching relays as result.

Further criteria brought this number down to 100.

We limited our search, as previously, to nodes above a certain bandwidth to avoid the (relative) fluctuations of smaller servers. We picked here $5 \mathrm{MiB} / \mathrm{s}$ as threshold (for reasons explained in further detail just below).

In addition to the already mentioned considerations that come with a choice of a 'proxy' (i.e. approximation), and to give the reader some more insights into the conditions in which this research took place:

- Fluctuations in bandwidth, small, occurred at least in two occasions we think.

- Two servers went offline in the middle of our research : one relay with an advertised bandwidth of $2 \mathrm{MiB} / \mathrm{s}$, followed by another of 4.75 $\mathrm{MiB} / \mathrm{s}$ (ISP : "DigitalOcean, LLC").

\section{Results}

\begin{tabular}{c|c|c} 
ISP & number of relays amongst 'oldest' & bandwith \\
Online & 7 & $143 \mathrm{MiB} / \mathrm{s}$ \\
Hetzner & 13 & $135 \mathrm{MiB} / \mathrm{s}$ \\
OVH & 10 & $84 \mathrm{MiB} / \mathrm{s}$ \\
XS4ALL & 2 & $40 \mathrm{MiB} / \mathrm{s}$ \\
Bahnhof & 1 & $38 \mathrm{MiB} / \mathrm{s}$ \\
myLoc & 2 & $26 \mathrm{MiB} / \mathrm{s}$
\end{tabular}

Table 1: 'oldest' Tor relays and commercial ISP's.

Alternatives to mainstream ISP's have come out of this research, in addition to their occurrences in our previous one : $X S 4 A L L$, a dutch ISP with a history of activism and defense of the Internet (similar to Swedish Bahnhof), hoster of two of the largest 'oldest' relays in the Tor network, and $m y L o c^{2}$ - to name a few.

\footnotetext{
${ }^{1}$ There were two bridges, ndnop2 and ndnop0 among results.

${ }^{2}$ https://trac.torproject.org/projects/tor/wiki/doc/ISPCorrespondence
} 
Relay calyx07 merits further comment : it is the only hosted by the Calyx Institute in Europe (amongst those registered here at least), where they chose $X S_{4} A L L$ for ISP.

Relay marcuse 1 and marcuse 2 belong to previously encountered association Nos oignons.

\begin{tabular}{c|c|c} 
University / group & relays amongst 'oldest' & bandwidth \\
University of Waterloo & gurgle & $40 \mathrm{MiB} / \mathrm{s}$ \\
MIT (CSAIL) & csailmitnoexit & $18 \mathrm{MiB} / \mathrm{s}$ \\
Stanford & everfailed & $12 \mathrm{MiB} / \mathrm{s}$ \\
University of Washington & UWashingtonCSE & $6 \mathrm{MiB} / \mathrm{s}$ \\
Boston University & BostonUCompSci & $5 \mathrm{MiB} / \mathrm{s}$ \\
UNC & ibibUNCO & $5 \mathrm{MiB} / \mathrm{s}$ \\
CMU & cmutornode & $3 \mathrm{MiB} / \mathrm{s}$
\end{tabular}

Table 2: universities and 'oldest' Tor relays.

One massive fact, recorded here, as previously, is the large, if not complete absence of European universities.

Where, in the Queen's name, are Cambridge and Oxford? Edinburgh? And, where ETHZ? And, all the rest? Such questions only fine gentlemen and gentlewomen can answer.

Social courage appears to not be - including geographically - unequally distributed.

(We do note however the presence of SUNET, the Swedish University Computer Network.)

\section{Addition : most resilient exit nodes}

To complement our previous research we provide the following additional data, results :

\begin{tabular}{c|c|c} 
University / group & exit nodes amongst 'most resilient' & bandwidth \\
University of Waterloo & gurgle & $40 \mathrm{MiB} / \mathrm{s}$ \\
Boston University & BostonUCompSci & $5 \mathrm{MiB} / \mathrm{s}$ \\
UNC & ibibUNCO & $5 \mathrm{MiB} / \mathrm{s}$ \\
CMU & cmutornode & $3 \mathrm{MiB} / \mathrm{s}$
\end{tabular}

Table 3: universities and 'most resilient' Tor exit nodes.

\section{Addition : decade old and still running}

Finally, we look at the very oldest, still running relays within the Tor network : those with a minimum recorded existence of 10 years. 


\begin{tabular}{|c|c|c|}
\hline Relay & ISP & First Seen \\
\hline che & Bahnhof AB & $2007-10-27$ \\
\hline $\begin{array}{l}\text { RazorsEdge } \\
\text { dao }\end{array}$ & $\begin{array}{l}\text { Satellite Management Services, Inc. } \\
\text { dao Consulting, LLC }\end{array}$ & $\begin{array}{l}2007-10-27 \\
2007-11-23\end{array}$ \\
\hline $\begin{array}{c}\text { Oxbadc0ffe } \\
\text { moses }\end{array}$ & $\begin{array}{c}\text { Deutsche Telekom AG } \\
\text { Linode. LLC }\end{array}$ & $\begin{array}{l}2008-02-17 \\
2008-05-15\end{array}$ \\
\hline DieYouRebelScum1 & Croatian Academic and Research Network & $2008-05-18$ \\
\hline Pasquino & eStruxture Data Centers Inc. & 2008-07-03 \\
\hline DigitalBrains & Xs4all Internet BV & $2008-07-13$ \\
\hline Hermes & MCI Communications Services ... & $2008-10-06$ \\
\hline
\end{tabular}

Table 4: Still running Tor relays with a recorded existence of a decade or more. 


\section{References}

—. 2019. "Fearless, 1000 days and still running : the 'most resilient' exit nodes of the Tor network and their ISP's - a quantitative approach." 
Ordered by most bandwidth to least* :

(*as in our previous research)

- - $50 \mathrm{MiB} / \mathrm{s}$

Lule

gurgle

che

fluxe4

Multivac

regar42

hviv104

Logforme

3cce3a91f6a625

BlickWinkel

-. $30 \mathrm{MiB} / \mathrm{s}$

DFRI0
Libero
torexit42
DFRI3
ndnop2
ndnop0
Jans
SEC6xFreeBSD64
dexter

-. $20 \mathrm{MiB} / \mathrm{s}$

hsjeufh24h6
Truie
Fastnet
TorMachine
csailmitnoexit
storm
AsiaArgento
ndnr1
Totonicapanp2
Cicolina
rehm
bauruine203
bauruine204
TangeNLV
DFRI1
DFRI4
birnenpfeffimitzimt
fluxe3
tortillero
everfailed
puertasecreta
informationalley
Finisterre
VERITAS
SweRaspiTor3
multisec2
lewwerDuarUesSlaav
niijo1
CalyxInstituteo7
multisec4

SUNET SUNET Swedish University Network

University of Waterloo

Bahnhof $A B$

Verein zur Foerderung eines Deutschen Forschungsnetzes e.V.

Online S.a.s.

Online S.a.s.

SURFnet bv

Telenor Norge AS

T-Mobile Thuis BV

Xs4all Internet BV

Foreningen for digitala fri- och rattigheter Specialized Bulletin Board Systems

Baytems Holdings 0y

Foreningen for digitala fri- och rattigheter

! na [NB. these are bridges]

! na [NB. these are bridges]

Init7 (Switzerland) Ltd.

SURFnet bv

Magyar Telekom plc.

myLoc managed IT AG

OVH SAS

Hetzner Online $\mathrm{GmbH}$

Hetzner Online $\mathrm{GmbH}$

Massachusetts Institute of Technology

Hetzner Online $\mathrm{GmbH}$

Online S.a.s.

UNINETT AS

Online S.a.s.

VNET a.s.

IP-Only Networks AB

Online S.a.s.

Online S.a.s.

Fiberby ApS

Foreningen for digitala fri- och rattigheter

Foreningen for digitala fri- och rattigheter

D-hosting die Rackspace \& Connectivity $\mathrm{GmbH}$

Hetzner Online $\mathrm{GmbH}$

Orange Espagne SA

Stanford University

Plusserver $\mathrm{GmbH}$

Hetzner Online $\mathrm{GmbH}$

Hetzner Online $\mathrm{GmbH}$

Massachusetts Institute of Technology

Telenor Norge AS

Broadnet AS

ServerAstra Kft.

Online S.a.s.

Xs4all Internet BV

Broadnet AS 


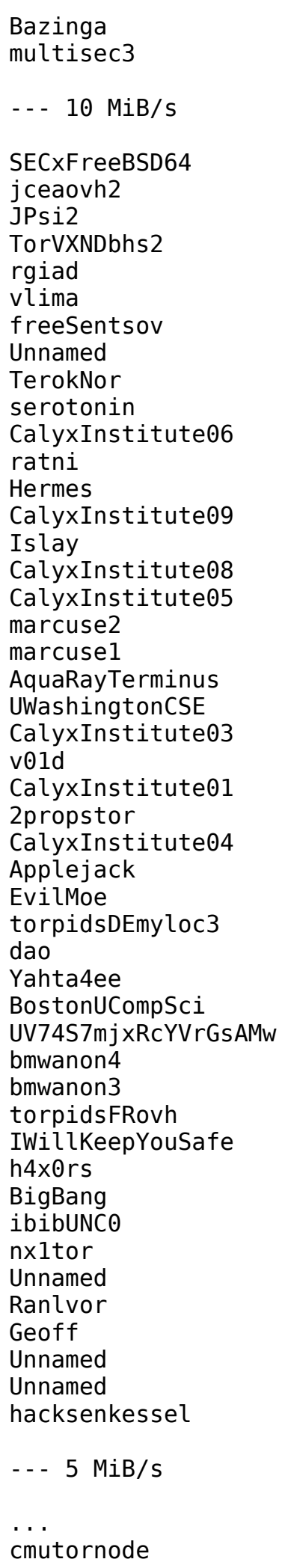

Host Europe $\mathrm{GmbH}$

Broadnet AS

SURFnet bv
OVH SAS
Host Europe GmbH

OVH SAS

RGnet, LLC

Greek Research and Technology Network S.A

Net By Net Holding LLC

OVH SAS

LeaseWeb Netherlands B.V.

Hetzner Online $\mathrm{GmbH}$

The Calyx Institute

OVH SAS

MCI Communications Services, Inc. d/b/a Verizon Business

The Calyx Institute

OVH SAS

The Calyx Institute

The Calyx Institute

Ielo-liazo Services SAS

Ielo-liazo Services SAS

Aqua Ray SAS

University of Washington

The Calyx Institute

VimpelCom

The Calyx Institute

Consolidated Communications, Inc.

The Calyx Institute

Hetzner Online $\mathrm{GmbH}$

OVH SAS

myLoc managed IT AG

dao Consulting, LLC

Hetzner Online $\mathrm{GmbH}$

Boston University

OVH SAS

Hetzner Online $\mathrm{GmbH}$

Hetzner Online $\mathrm{GmbH}$

OVH SAS

Broadnet AS

Hetzner Online $\mathrm{GmbH}$

Level 3 Parent, LLC

University of North Carolina at Chapel Hill

NEXCUS TECHNOLOGIES LLC

Hurricane Electric LLC

Hetzner Online $\mathrm{GmbH}$

EVANZO e-commerce $\mathrm{GmbH}$

I.C.S. Trabia-Network S.R.L.

OVH SAS

Host Europe $\mathrm{GmbH}$

Carnegie Mellon University 